\title{
Vom Schreibtisch in den virtuellen Kursraum
}

\author{
Die Akademie für Praxis und Wissenschaft (APW) macht das Lernen leichter: Im APW-Kursraum stehen für \\ jeden gebuchten Präsenzkurs umfangreiche digitale Zusatzangebote zur Verfügung.
}

O wird das Lernen deutlich leichter und der Griff zu Lehr$\checkmark$ materialien ein Kinderspiel: Für die bestmögliche Betreuung und Unterstützung vor, während und nach dem Besuch einer ihrer Fortbildungsangebote stellt die APW in virtuellen geschlossenen Kursräumen ein umfangreiches digitales Serviceangebot kostenfrei zur Verfügung. Sobald eine Kursbuchung eingegangen ist, hat der Teilnehmer Zugang zum geschlossenen APWKursraum der gebuchten Veranstaltung.

\section{Hier steht eine Anzeige.} Springer

\section{Hier steht eine Anzeige.}

\author{
Springer
}

Ein Beispiel mit Screenshot:

So sieht er aus, der virtuelle Klassenraum.

\section{Was ist ein virtueller Kursraum?}

Im Grunde funktioniert ein APW-Kursraum ähnlich wie ein Klassenzimmer. Hier treffen die Lernenden virtuell zusammen, können sich austauschen und dem Referenten Fragen zum Lernstoff stellen. Außerdem findet sie hier eine Auswahl an Fachmedien, die den während der Präsenzveranstaltung vermittelten Lehrstoff ergänzen können. Ganz gleich, ob ein APW-Curriculum, ein Einzelkurs oder eine der Fachtagungen gebucht werden - mit dem digitalen Serviceangebot wird der Teilnehmer effektiv beim Lernen unterstützt - und zwar weit über die eigentliche Veranstaltung hinaus.

\section{Die APW-Kursräume helfen auf unterschiedliche Weise}

Die virtuellen Kursräume ermöglichen es, mit den Referenten und den übrigen Kursteilnehmern in Kontakt zu treten. Nutzer haben die Möglichkeit, den Referenten im Vorfeld der Veranstaltung Fragen zu stellen. Im Anschluss an die Veranstaltung können sie sich mit den übrigen Kursteilnehmern vernetzen und gemeinsam den vermittelten Stoff diskutieren und vertiefen. Hierzu gibt es in jedem Kursraum ein eigens eingerichtetes Diskussionsforum.

Um den während des besuchten Kurses vermittelten Lernstoff zu vertiefen, stellt die APW in dem jeweiligen Kursraum jeweils zwei digitale Medienangebote der kooperierenden Fachverlage - Quintessenz-Verlag, Springer Zahnmedizin und Deutscher Ärzteverlag - kostenfrei zur Verfügung. Die bereitgestellten Medien stehen in einem engen Zusammenhang mit der besuchten Fortbildung und bieten einen zusätzlichen Mehrwert für das Verständnis. Darüber hinaus finden sich hier wertvolle Abrechnungstipps sowie - sofern vorhanden - thematisch passende Leitlinien, Stellungnahmen und Mitteilungen der DGZMK und ihrer assoziierten Gesellschaften.

\section{Dokumentenablage und Archivierung}

Die Kursskripte werden im APW-Kursraum eingestellt. Alle Referenten wurden gebeten, ihre Kursskripte zur digitalen Bereitstellung im APW-Kursraum verfügbar zu machen. So kann der Teilnehmer sich während der Veranstaltung ganz auf die Vorträge der Referenten konzentrieren. Sollte einmal die Anfahrtsskizze nicht zu finden sein, genügt ebenfalls ein Blick in den virtuellen Kursraum. Hier können auch bequem Rechnungen eingesehen, heruntergeladen und ausgedruckt werden.

Für weitere Fragen und Anregungen können Interessenten sich an die Portalmanagerin Kirstin Petzold unter der Telefonnummer 0211 669673-30 oder via E-Mail an dgzmk.petzold@ dgzmk.de wenden.

(Markus Brakel) 